\title{
Frühe Menopause erhöht Risiko für Lungenerkrankungen
}

\author{
Frühe Menopause sowie Verlust von Gebärmutter \\ und Ovarien sind assoziiert mit einem akzelerierten \\ Abfall der Lungenfunktion.
}

Beim europäischen Lungenkongress ERS 2016 berichteten Autoren des Imperial College in London über eine von der British Lung Foundation unterstützten Studie mit 140.000 Frauen, bei denen sowohl Daten über die Lungenfunktion als auch Informationen über den Menopausen-Status vorlagen. Sie untersuchten Assoziationen zwischen den Lungenfunktionsparametern $\mathrm{FEV}_{1}, \mathrm{FVC}$, der spirometrischen Restriktion $(\mathrm{FVC}<\mathrm{LLN})$ sowie der Atemwegsobstuktion $\left(\mathrm{FEV}_{1} / \mathrm{FVC}<\mathrm{LLN}\right)$, setzten dies in Beziehung zum menopausalen Status bzw. dem Alter bei der Menopause und schlossen den Einfluss des Rauchens statistisch aus.

In der „mit Abstand größten Studie zu dieser Fragestellung“ stellte sich heraus, dass Frauen schon mit einer natürlichen Menopause einen deutlichen Verlust der Lungenfunktion erfahren. Ihr FVC war im Schnitt um $42 \mathrm{ml}$ niedriger und ihr FEV $\mathrm{F}_{1}$ im Schnitt um 34 $\mathrm{ml}$ niedriger als bei prämenopausalen Frauen. Die Wahrschein-

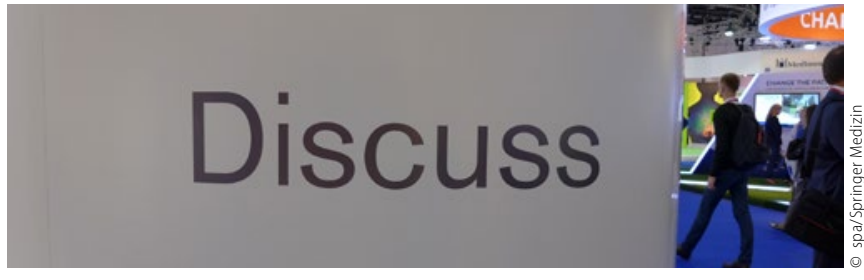

Welche praktische Konsequenz aus einem Studienergebnis zu ziehen ist, lässt sich freilich oft trefflich diskutieren.

lichkeit für eine im Altersdurchschnitt niedrigere Lungenfunktion war um 27\% erhöht, nach Hysterektomie um 31-36\%.

Frauen mit einer Menopause vor dem 45. Lebensjahr wiesen im Alter über 60 Jahren ein um 20\% erhöhtes Risiko für eine erniedrigte Lungenfunktion auf als Frauen mit einer Menopause im Alter von 48-53 Jahren.

Dr. med. Dirk Einecke

Quelle: International Congress 2016 der European Respiratory Society (ERS), London, 3.-7. September 2016

\section{Therapie der schweren COPD mit nur einem Hub täglich}

In der FULFIL-Studie war eine ICS/LABA/LAMA-Fixkombination einer dualen ICS/LABA-Kombi bei schwerer COPD überlegen. Beim ERS-Kongress wurden mit TRILOGY, TRINITY und FULFIL 3 große Phase-3-Studien zu Fixkombinationen eines inhalativen Steroids (ICS) plus 2 Bronchodilatatoren (LABA/LAMA) bei schwerer COPD vorgestellt. Alle 3 trafen ihre primären Endpunkte, sie waren Therapien mit 1 (LAMA) oder 2 Arzneien (ICS/LABA) überlegen.

In der doppelblinden FULFIL-Studie war eine Fixkombination der 3 lang wirksamen Arzneien von GSK Fluticasonfuroat, Umeclidinium und Vilanterol (FF/UMEC/VI 100/62,5/25 $\mu \mathrm{g}$ 1x/d) mit Budesonid/Formoterol (400/12 $\mu \mathrm{g}$, $2 x / d$, Symbicort ${ }^{\oplus}$ Turbohaler $^{\oplus}$ von AstraZeneca) verglichen worden. Der wesentliche Unterschied bestand in dem zusätzlichen LAMA Umeclidinium. Ein weiterer ist die längere Wirkdauer. FF/UMEC/VI wird nur 1x/d gegeben. Dies mag ein Compliancevorteil vs. dem 2x/d zu dosierenden Budesonid/Formoterol sein. Auch die in TRILOGY und TRINITY getestete fixe Dreierkombi von Chiesi (Beclomethason-Dipropionat, Formoterol-Fumarat, GlycopyrroniumBromid, BDP/FF/GB) wird 2x/d appliziert. An FULFIL nahmen 1.810 Patienten mit symptomatischer COPD und einer $\mathrm{FEV}_{1}<50 \%$ oder von $50-80 \%$ und 2 mittelschweren bis schweren Exazerbationen im Vorjahr teil. 2 co-primäre Endpunkte waren Lungenfunktion ( $T a l-F E V_{1}$ ) und Lebensqualität (SGRQ-Score nach 24 Wochen). Wichtigster sekundärer Endpunkt war die Rate mittelschwerer/schwerer Exazerbationen in 24 Wochen. Mit Tripletherapie hatten die Patienten nach 24 Wochen eine deutlich bessere Lungenfunktion als nach ICS/ LABA, die FEV , war $171 \mathrm{ml}$ höher. Auch die Lebensqualität war signifikant besser: 50\% (Triple) vs. 41\% (Dual) der Patienten besserten sich im SGRQ um mind. 4 Punkte. Patienten mit Tripletherapie hatten in 24 Wo. um 35\%, nach 1 Jahr $44 \%$ weniger Exazerbationen. Verträglichkeit $u$. Nebeneffekte waren vergleichbar. Dass ICS/LABA/LAMA vs. ICS/LABA überlegen ist, überrascht nicht, angesichts der Wirkstärke des LAMA, so Prof. Claus Vogelmeier, Marburg, zu FULFIL und TRILOGY. Interessant wird der Vergleich mit der dualen Bronchodilatation LABA/LAMA. Er wird zeigen, wer ICS tatsächlich braucht. GSK und Chiesi haben Studien dazu aufgelegt, Ergebnisse werden 2018 erwartet. de

Quelle: ERS-Kongress, London, 3.-7. September 2016

\section{Schwere COPD: Subgruppe profitiert von Tripletherapie}

Es scheint eine kleine Gruppe von COPD-Patienten zu geben, die neben Bronchodilatatoren inhalative Steroide benötigen: Solche mit besonders häufigen Exazerbationen und hohen Eosinophilen-Zahlen.

Pneumologen beklagen häufig eine Übertherapie mit inhalativen Steroiden (ICS) bei COPD. Gemäß Leitlinie sind sie nur bei COPD indiziert, die häufig exazerbiert. Tatsächlich erhalten aber fast ein Drittel aller COPD-Patienten ICS, unabhängig vom COPD-Stadium. Doch ob die häufigen Exazerbierer ICS wirklich benötigen, wurde jüngst von zwei Studien in Frage gestellt. Dies war zum einen die FLAME-Studie (Wedzicha JA, et al. N Engl J Med 2016; 374:2222-34). Diese verglich - bei 3.362 COPD-Patienten mit häufigen Exazerbationen - eine ICS/LABA-Kombination mit einer LABA/LAMA-Kombination. Es stellte sich heraus, dass die Gruppe ohne ICS an Bord signifikant weniger Exazerbationen erlitten. Die andere Studie war die WISDOM-Studie (Magnussen H, et al. N Engl J Med 2014;371:1285-94). Hier wurde bei 2.485 COPD-Patienten und wenigstens einer Exazerbation im Vorjahr unter Tipletherapie mit ICS, LABA und LAMA das ICS langsam in der Dosis reduziert und schließlich abgesetzt. Es zeigte sich, dass sich das Exazerbationsrisiko dadurch nicht erhöhte gegenüber Fortführung der Triple-Therapie.

Beim ERS -Kongress präsentierte Prof. Peter Calverley von der Universität Liverpool nun eine neue Substudie der WISDOM-Studie. Die Autoren gingen in einer Post-hoc-Analyse der Frage nach, ob bei besonders schwerer COPD das Absetzen des ICS doch nachteilhaft ist. Sie konzentrierten sich dabei auf zwei Risikomarker: häufige Exazerbationen ( $>2 / J$ ahr) und hohe Eosinophilen-


jährlich mehr als zwei Exazerbationen und hohen Eosinopilen-Zahlen das ICS doch benötigten. Setzten sie es ab, stieg das Exazerbationsrisiko signifikant an. Auch die Lungenfunktion verschlechterte sich. Besonders deutlich zeigte sich dies für Patienten mit $>400$ Eosinophilen/ $/$ l. „Diese Daten helfen, eine kleine, aber wichtige Patientengruppe zu definieren, bei denen künftig die Tripletherapie ICS/LAMA/LABA prospektiv getestet werden sollte", resümierte Calverley.

Quelle: ERS-Kongress, London, 3.-7. September 2016 